\title{
dspace.vutbr.cz
}

\section{Low-pressure twin-fluid atomization: Effect of mixing process on spray formation}

ZAREMBA, M.; WEIß, L.; MALÝ, M.; WENSING, M.; JEDELSKÝ, J.; JÍCHA, M.

International Journal of Multiphase Flow

2017, vol. 89, March 2017, pp. 277-289

ISSN: 0301-9322

DOI: https://doi.org/10.1016/j.ijmultiphaseflow.2016.10.015

Accepted manuscript

(C) 2017. This manuscript version is made available under the CC-BY-NC-ND 4.0 license (http://creativecommons.org/licenses/by-nc-nd/4.0/), doi:

https://doi.org/10.1016/j.ijmultiphaseflow.2016.10.015

Final version available from https://www.sciencedirect.com/science/article/pii/s0301932216300271 


\title{
Low-Pressure Twin-Fluid Atomization: Effect of Mixing Process on Spray Formation
}

\author{
Authors affiliation \\ Matouš Zaremba ${ }^{1}$, Lukas Weiß $\aleph^{2,3}$, Milan Malý1, Michael Wensing ${ }^{2,3}$, Jan Jedelský1 ${ }^{1}$, Miroslav Jícha ${ }^{1}$ \\ ${ }^{1}$ Brno University of Technology, Faculty of Mechanical Engineering, Department of the \\ Thermodynamics and Environmental Engineering, Czech Republic \\ ${ }^{2}$ Friedrich Alexander Universität, Institute of Engineering Thermodynamics, Erlangen-Nuremberg, \\ Germany \\ ${ }^{3}$ Erlangen Graduate School in Advanced Optical Technologies, Erlangen, Germany
}

Corresponding author: zaremba@fme.vutbr.cz

\section{Key words}

low-pressure; atomization; twin-fluid; droplet dynamics; liquid breakup; back illumination

\section{Abstract}

The present work comparably examines four different twin-fluid atomizers operated under the same operating conditions. Spray formation was examined by several approaches. The internal flow pattern was estimated using a simplified analytical approach, and the results were supported by the observation of the liquid discharge in the near-nozzle region. A high-speed back illumination was used for visualisation of the primary breakup. In the region of fully developed spray, the dynamics of droplets was studied using a phase-Doppler analyser (PDA). The information obtained from all methods was then correlated. Results show that the spray formation process depends mainly on the internal design of twin-fluid atomizer at low gas to liquid ratios (GLR). The amount of gas influences the character of the internal two-phase flow, a mechanism of the liquid breakup, droplet dynamics and a resulting drop size distribution. Differences among the atomizers are reduced with the increase in GLR. Moreover, it was shown that a certain mixing process can inherently create the annular internal flow which generates a stable spray characterized by relatively low mean droplet size.

\section{Introduction}

Twin-fluid atomization is a method for spraying of liquids using an additional medium - pressurized gas that enhances the liquid breakup. Twin-fluid atomizers are common in various industrial applications, e.g. liquid fuel spray, drying in food processes, etc. According to the mixing process, the twin-fluid atomizers can be divided into internal-mixing and external-mixing types. This work focuses on the variations of the internal-mixing nozzles.

Featured designs of internal-mixing atomizers have been examined in detail, such as the effervescent atomizer (Sovani et al., 2001; Konstantinov et al., 2010) or the Y-Jet atomizer (Mullinger and Chigier, 1974; Song and Lee, 1996; Pacifico and Yanagihara, 2014). It was proved that 
effervescent atomizers can operate at relatively low-pressures compared to conventional pressure atomizers, and at a low consumption of gas in comparison with air-blast atomizers as noted in Sovani et al., (2001). Moreover, they are relatively insensitive to rheological parameters of liquid due to a specific mechanism of liquid breakup - bubble explosions (Konstantinov et al., 2010). This breakup mechanism is conditional by the bubbly internal two-phase flow. When this two-phase flow passes through the exit orifice, the gas bubble expands rapidly and shatters the liquid into the smaller fractions. Mullinger and Chigier, (1974) showed that the liquid breakup mechanism in the Y-jet atomizer is relatively insensitive to the operating regime. Because of these advantages, together with a simple construction and large exit orifices, these techniques are now frequently used in industry; the main domain of their use is spraying of highly viscous liquid fuels for combustion applications (Konstantinov et al., 2010).

Formation of the spray generated by an internal-mixing twin-fluid atomizer begins inside its body. The internal flow determines the way the liquid disintegrates and how the spray develops in time and space. The character of the internal two-phase flow depends mainly on the gas to liquid ratio (Konstantinov et al., 2010) and, as noted in Mlkvik et al., (2015), on the internal design of the atomizer. Main features of the internal design are the diameter of mixing chamber, the number of aerator holes and the way the fluids mix inside (Gadgil and Raghunandan 2011a; Jedelsky et al., 2009). Further parameters, such as the operating pressure ( $\mathrm{Li}$ et al., 2012) and fluid's physical properties, have a lesser influence on the flow pattern (Liu et al., 2011; Lund et al., 1993; Stähle et al., 2015a).

The internal two-phase flow was examined by several approaches: The experiments were conducted using transparent atomizers (Song and Lee, 1996; Huang et al., 2008; Li et al., 2012; Ju et al., 2015; Stähle et al., 2015a). These studies confirmed the hypothesis of transition from a bubble flow (low-GLR) to a plug flow and further to the annular flow (high-GLR). However, an experimental approach is not always possible so numerical simulations, theoretical two-phase flow maps (Jedelsky and Jicha, 2008) or a simplified description based on liquid/gas momentum ratio have been used to estimate the internal flow patterns (Song and Lee, 1996; Mlkvik et al., 2015).

The relation between the internal two-phase flow and the primary breakup mechanism was investigated in several publications, e.g. (Buckner and Sojka, 1991; Santangelo and Sojka, 1995; Catlin and Swithenbank, 2001; Gadgil and Raghunandan, 2011b). At low-GLR regimes, the breakup is based on the gas bubble explosions. When a gas bubble passes through the exit orifice, it expands in both radial and axial direction. It means that the liquid film, which surrounds the gas, is stretched out which causes film thinning. As the GLR increases, and consequently also the gas mass flow, the bubbles become larger, and the thickness of the liquid film is reduced. This forms a shape similar to an annular liquid sheet, which results in the creation of the so-called tree-like structures producing individual droplets (Santangelo and Sojka, 1995; Sutherland et al., 1997).

Even though there is a sufficient number of publications aimed at the breakup of basic liquid structures (streams, liquid sheets or single droplet breakup), e.g. (Dumouchel, 2008; Eggers and Villermaux, 2008), a detailed description of such a complex process as the effervescent atomization has not been previously made (Konstantinov et al., 2010). Studies which describe the breakup lowpressure regimes are still relatively rare, and a deeper understanding of the spray formation is needed. Moreover, the effect of different atomizer designs on liquid breakup and spray properties has been studied only in several cases, e.g. (Stähle et al., 2015b). Therefore, it is difficult to choose and design the atomizer for a specific application (Ferreira et al., 2009b; Diego-Marin et al., 2009; Mujumdar et al., 2010).

It was shown that at low-pressure and low-GLR regimes the design of atomizers plays an important role Mlkvik et al., (2015). The mixing process heavily influences the character of the internal twophase flow at low-GLR and low-pressure regimes. It was revealed that the atomization efficiency, defined as the ratio of energy to increase the surface area of the liquid to the energy applied to the spraying atomizer (Bayvel and Orzechowski, 1993), grows when the operating pressure and GLR decrease (Jedelsky and Jicha, 2013). The relation between the atomization efficiency and GLR has an 
approximately inverse logarithmic tendency. A similar relationship was also found between the efficiency and pressure. Thus the low-GLR and low-pressure regimes are considered as highlyeffective. Therefore, it is important to comparably examine various designs of atomizers under these conditions.

The present paper examines four different internal-mixed atomizers. These are the two common types: the Y-jet atomizer and the "outside in gas" effervescent atomizer (OIG). Other two atomizers are newly developed ones: specific construction of the "outside in liquid" effervescent atomizer (OIL) and the atomizer which was designed on the basis of previous designs of twin-fluid atomizers by workers: Chin (1995), Ferreira e al., (2001) and Tamaki et al., (2004), so-called CFT atomizer. All four atomizers were studied in the previous work of Mlkvik et al., (2015) where a mixing process and an analysis of breakup of liquid structures (ligaments) were described for several different liquid viscosities. However, due to limited optical access, the study of the liquid breakup mechanism has not yet been conducted. Moreover, we examined the atomizers at lower pressures, half the magnitude of the minimal pressure examined in Mlkvik et al., (2015), and lower GLR, from 2.5\% to 20\%. The lowGLR regimes were not fully described because the atomizer's exit orifices were blocked due to the crystallization of the liquid and no spray was generated. Thus we could extend the previous description by studying the influence of the internal design on the liquid breakup and discuss its effect on the spray characteristics. Our aim is to examine various designs of twin-fluid atomizers under highefficient regimes and to point out the aspects influencing the spray characteristics. Due to the opaque body of atomizers, an assumption of the internal flow was made by a simplified analytical approach based on findings of Song and Lee, (1996) and Mlkvik et al., (2015). We used a high-speed camera with a long-distance microscope for examination of the liquid breakup. Droplet dynamics was analysed using the data from the PDA system. In this paper, the results are chronologically ordered according to the spray formation in internally-mixed twin-fluid atomizers.

\section{Experimental setup}

Atomizers were operated on a cold test bench at a room temperature of $23{ }^{\circ} \mathrm{C}$ and still ambient conditions. A detailed description of the test bench can be found in Jedelský et al.,(2014). Table 1 shows the measured values of operating regimes. The pressure chosen was $70 \mathrm{kPa}$ and GLR from 2.5 to $20 \%$. In twin-fluid atomizers, GLR is calculated by dividing the liquid and gas mass flows. It is defined as the ratio of mass flowrate of atomizing gas through the atomizer to that of liquid (Sovani et al., 2001). We then can express the GLR in percentages, which shows how much of the total mass flow is occupied by the gas. To control the GLR value during the measurement, we used a mass flow meter for liquid (combined uncertainty of measured value $1 \%$ for a confidence level of $95 \%$ ), mass flow meter for gas (5\%) and pressure sensors (0.4\%).

Table 1. Parameters of operating regimes.

\begin{tabular}{|l|cccc|}
\hline Name & $\begin{array}{c}p_{L} \\
{[\mathrm{kPa}]}\end{array}$ & $\begin{array}{c}p_{G} \\
{[\mathrm{kPa}]}\end{array}$ & $\begin{array}{c}m_{L}\left[\mathrm{~kg} \cdot \mathrm{h}^{-}\right. \\
1]\end{array}$ & $\begin{array}{c}G L R \\
{[\%]}\end{array}$ \\
\hline \multirow{5}{*}{ CFT } & 71 & 70 & 4.30 & 2.4 \\
& 70 & 70 & 3.04 & 5.0 \\
& 70 & 70 & 1.88 & 10.2 \\
& 71 & 71 & 1.24 & 20.2 \\
\hline \multirow{5}{*}{ OIG } & 70 & 70 & 4.08 & 2.5 \\
& 70 & 69 & 2.65 & 5.0 \\
& 67 & 70 & 1.70 & 10.2 \\
& 69 & 70 & 1.14 & 19.8 \\
\hline \multirow{5}{*}{ OIL } & 69 & 71 & 3.75 & 2.5 \\
& 68 & 69 & 2.55 & 5.1 \\
& 72 & 71 & 1.72 & 10.3 \\
& 71 & 72 & 1.12 & 20.8 \\
\hline
\end{tabular}




\begin{tabular}{|c|cccc|}
\hline & 74 & 71 & 3.49 & 2.5 \\
Y-jet & 71 & 71 & 2.54 & 5.2 \\
& 69 & 69 & 1.68 & 10.2 \\
& 69 & 71 & 1.11 & 20.4 \\
\hline
\end{tabular}

We assume that the selected atomizers were designed for liquid fuel combustion; thus we used light heating oil (LHO), and pressurized air as the tested fluids. Physical properties of the fluids are documented in Table 2.

Table 2. Physical properties of fluids at room temperature.

\begin{tabular}{|c|ccc|}
\hline Fluid & $p\left[\mathrm{~kg} / \mathrm{m}^{3}\right]$ & $\mu[\mathrm{kg} /(\mathrm{m} \cdot \mathrm{s})]$ & $\sigma\left[\mathrm{kg} / \mathrm{s}^{2}\right]$ \\
\hline LHO & 874 & 0.0185 & 0.0297 \\
Air & 1.23 & $1.81 \cdot 10^{-5}$ & \\
\hline
\end{tabular}

\subsection{Atomizers}

A detailed description of selected atomizers can be found in Mlkvik et al., (2015). In internal-mixing twin-fluid atomizers, the gas and liquid are mixed in the mixing chamber. Then this two-phase mixture flows downstream through the atomizer. As it passes through the exit orifice, the liquid breaks up due to the aerodynamics forces and a rapid expansion of the gas enhances the liquid breakup in the nearnozzle region.

Selected atomizers are schematically shown in Fig. 1. These designs have demonstrably different mixing processes but similar main dimensions. They were designed to have a similar hydraulic resistance under the same operating regimes, i.e. at the same pressure and GLR.

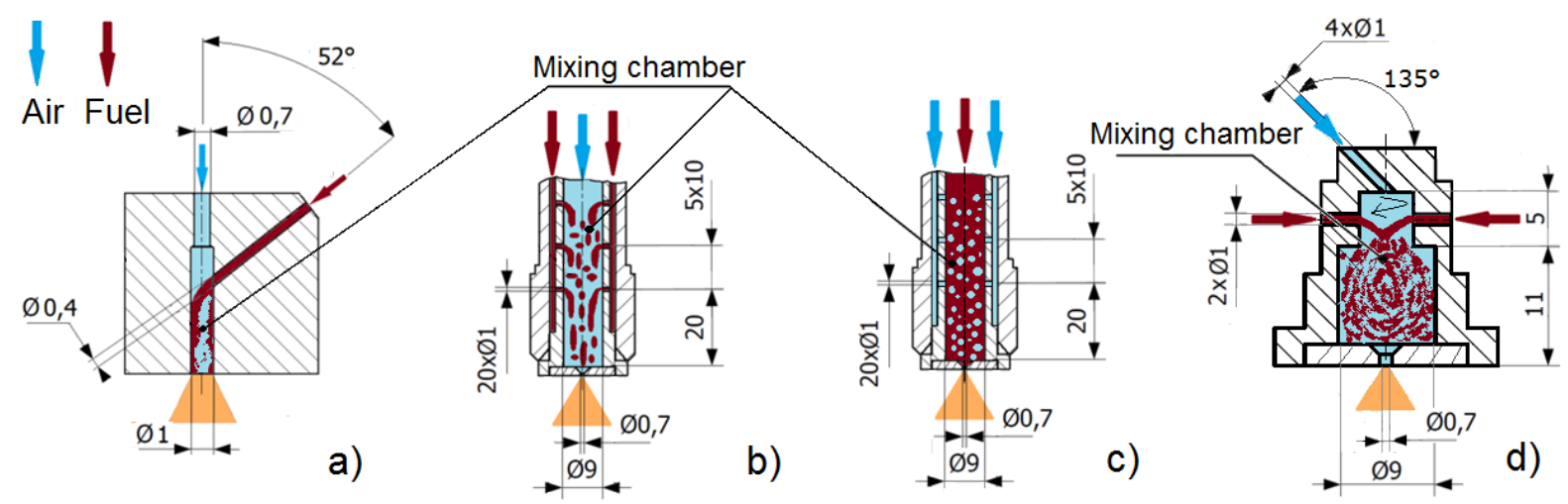

Fig. 1 Atomizers (figures are not drawn to the same scale), a) Y-jet, b) OIL, c) OIG, d) CFT.

\subsection{Measuring and visualisation techniques}

A back illumination technique was performed to record the liquid breakup in a near-nozzle region. The area of interest was illuminated by a pulse laser Cavilux HF System $810 \mathrm{~nm} / 500 \mathrm{~W}$ with laser pulse duration of 50 ns. We used a high-speed camera Photron FASTCAM SA-Z with long-distance macroscopic objective NAVITAR $12 \mathrm{X}$ with $0.25 \mathrm{X}$ attached lens and $1 \mathrm{X}$ F-mount adapter to capture the liquid breakup. The camera frame rate was 42,000 fps, and the resolution of images was $480 \times 1,024$ pixels. The area of interest, after trimming of recorded pictures, had dimensions of $7 \times 4$ $\mathrm{mm}$. A schematic layout of the experimental setup can be seen in Fig. 2. 


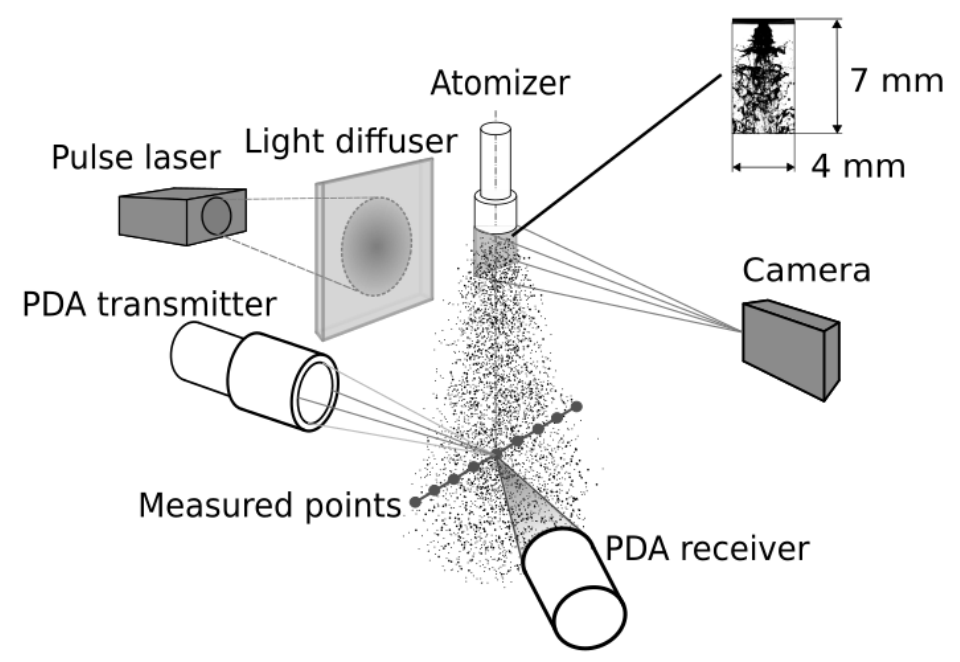

Fig. 2 Schematic layout of the experimental setup.

A fibre based 2D phase Doppler analyser was used to obtain time-resolved data about the droplet size and velocity. The atomizer was mounted on a traverse system. The main axis of the atomizer was set perpendicularly to the main axis of the transmitting optics of the PDA system. Measurements were performed at an axial distance of $100 \mathrm{~mm}$ downstream from the atomizer. The receiver optics was set to an angle of $69^{\circ}$ to the transmitter axis where the first scattering mode is dominant as recommended by the manufacturer of the PDA system. We measured 15 points across the spray profile by the step of $3 \mathrm{~mm}$. The system was set to measure 20,000 samples for at least one minute in low dense regions. A detailed description of the PDA system is available in Jedelský et al., (2014).

\section{Data processing}

This section describes the methods of how we estimated the character of the internal flow, how was done the post-processing of high-speed back illumination images and analysis of PDA data.

\subsection{Internal flow estimation}

Due to the opaque body of atomizers, we used a simplified analytical approach to estimate the internal flow pattern. This method was used in Mlkvik et al., (2015) and Song and Lee, (1996) and it compares momentum ratios of liquid and gas entering the mixing chamber. The liquid/gas momentum ratio $(\Phi)$ was calculated as follows:

$$
\Phi=\frac{m_{L}^{2}}{m_{G}^{2}} \cdot \frac{\rho_{G}}{\rho_{L}} \cdot \frac{d_{L}^{2}}{d_{G}^{2}} \cdot \sin \theta,
$$

where $m_{L}^{2}$ is the liquid mass flux per surface unit expressed in $\mathrm{kg} /\left(\mathrm{m}^{2} \cdot \mathrm{s}\right), d$ is a diameter of the channel which leads to the mixing chamber, see Fig. 1. In the case of multiple channels, which feed the liquid to the mixing point, a diameter $d$ is an equivalent diameter of the entire injection area, $\rho$ is fluid density and $\theta$ is an intersecting angle; the angle under which fluids are injected into the mixing chamber (for atomizers Y-jet, OIL, OIG and CFT are these angles $52^{\circ}, 90^{\circ}, 90^{\circ}, 135^{\circ}$ respectively, see Fig. 1). The subscript $L$ is related to the liquid phase and the subscript $G$ to the gas phase.

\subsection{Image post-processing}

The recordings from high-speed back illumination were evaluated using the following procedure: to remove the background noise, the raw image was contrast adjusted and filtered, Fig. 3 a). These processed images were used for visual evaluation of the liquid breakup. Further image post-processing 
was done using PCO Picture Viewer ${ }^{1}$. Mean images were calculated to evaluate a breakup length and optical spray density over the time, Fig. $3 \mathrm{~b}$ ). The pixel intensities in the mean image are the average of all pixel intensities of the loaded normal images at the same location (sum of the pixel intensities divided by the number of opened normal images). For better visibility of the spray contours, the image was colour-scaled according to light intensity into red, green, blue (RGB) spectrum, Fig. 3 c).

In processed figures, a dark blue colour indicates the minimum light intensity that corresponds to high spray density i.e. light was blocked by the spray. Decreasing the spray density is shown using the transition from blue to green and red. Dark red colour usually represents the edge of the spray, low dense regions (Jedelsky and Jicha, 2012). For evaluation of spray fluctuations and instabilities during the liquid breakup, root mean square (RMS) images were created, as shown in Fig. 3 d). The pixel intensities in the RMS image are computed by calculating the standard deviation of the pixel intensities at the same location in all normal images. RMS figures were again turned into the RGB colour spectrum, Fig. 3 e). RMS images show differences among the examined sequences. Thus a high intensity (red colour) indicates the positions of rapid variations of liquid and vice versa.

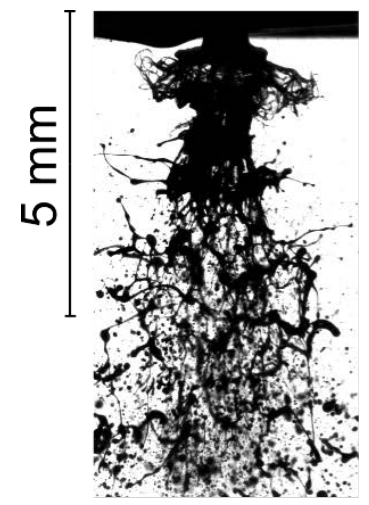

a)

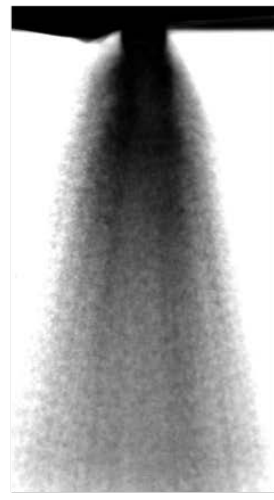

b)

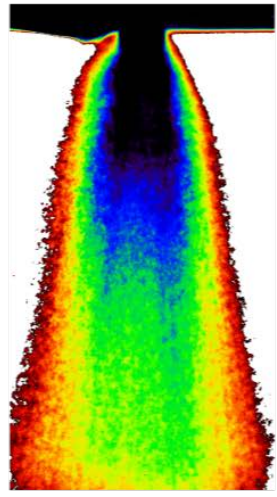

c)

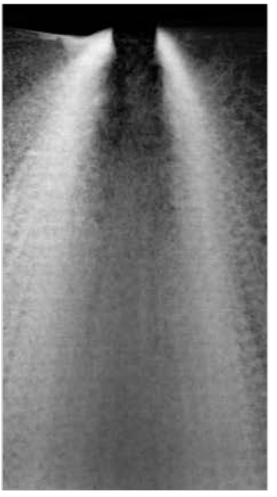

d)

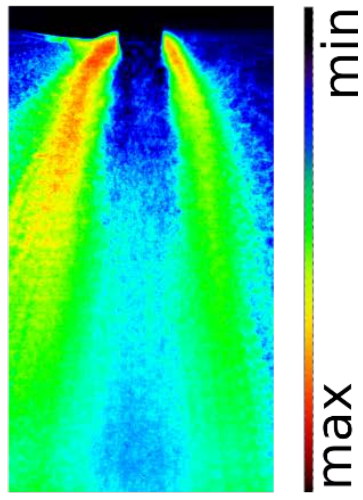

e)

Fig. 3 Steps of image post-processing: a) instantaneous image, b) mean image, c) mean image colour-scaled in RGB, d) RMS image, e) RMS image colour-scaled in RGB.

\subsection{PDA data post-processing}

To characterize a global spray quality, we used the integral value of Sauter mean diameter (ID32) which is often used in the evaluation of sprays designed for combustion applications. The derivation of ID32 is present in the appendix 2 of Jedelsky et al.,(2009) and the final equation, as used for our calculations, is shown below:

$$
I D 32=\frac{\sum_{i=2}^{n}\left(r_{i} \cdot D_{30, i}^{3} \cdot f_{i}\right)}{\sum_{i=2}^{n}\left(r_{i} \cdot D_{20, i}^{2} \cdot f_{i}\right)},
$$

where $r$ is the radial distance from the centre of the spray, $D$ is a droplet diameter and $f$ is a mean data rate (number of measured droplets per second) at a given point.

A description of particle behaviour in a gas flow was made by Stokes number (Stk). According to the definition, the Stk is a ratio of the characteristic time of a particle to a characteristic time of the flow. We used its modification for the sprays applications (Jedelsky and Jicha, 2014):

$$
S t k=\frac{\rho_{L} \cdot D^{2} \cdot\left|\left(U_{d}-U_{G}\right)\right|}{18 \cdot \mu_{G} \cdot Z},
$$

where $Z$ is decay constant, $U$ is velocity and $\mu_{G}$ is gas dynamics viscosity. Subscript $d$ is related to the measured droplets. The PDA system was used to measure droplet velocity and size. The gas velocity

${ }^{1}$ PCO Pictures, Doc Schneider Engineering, CH-8308 Illnau, Switzerland. 
was not measured directly. We assume that the smallest droplets (typically smaller than 5 microns) follow the motion of gas smoothly due to their low momentum. This approach was used in the previous publications to calculate Stokes numbers for droplets when there was no direct measurement of gas velocity, e.g. (Santolaya et al., 2010).

\section{Results and discussions}

This section is divided into several parts covering chronologically the fluid dynamics aspects of twinfluid atomization examined for the selected nozzles. As mentioned earlier, the liquid breakup starts inside the atomizer by mixing of the liquid and gas phases. The next stage of the spray formation, external to the atomizer, is the liquid breakup which is described using the recordings from the highspeed back illumination. In the region of fully developed spray, we used PDA from which the droplet dynamics was analysed. In the final stage, the atomizers are compared quantitatively.

\subsection{Estimation of the internal flow pattern}

The estimation of the internal flow pattern is based on the approach presented by Mlkvik et al., (2015) and Song and Lee, (1996). However, the following description is limited to the selected atomizers and might give just an insight into the assumption of the internal flow character. We describe the behaviour by a change in the ratio between the liquid and gas momentum $\Phi$ calculated according to equation 1, in section 3.1. Firstly, we comment on general aspects of the influence of $\Phi$ on the formation of the internal flow. Secondly, we describe the mixing processes of each atomizer individually.

\subsubsection{General aspects of the mixing processes}

We analyse the behaviour of the internal two-phase flow by the change of the liquid/gas momentum ratio $\Phi$ calculated according to equation 1 . If the ratio is low $(\Phi<1)$, the liquid inside does not have a sufficient momentum to penetrate into the centre of the mixing chamber; it should remain on its walls, as noted in Song and Lee, (1996). Generally, these low values of $\Phi$ occur in the case of high-GLR for all atomizers and also for all regimes of the OIL atomizer, see Table 3. The low momentum of liquid leads to the formation of the annular internal flow; the liquid is concentrated on the walls of the mixing chamber whereas the gas creates a continuous central core.

When the momentum ratio increases $(1<\Phi<3)$, the liquid should penetrate into the centre of the mixing chamber. This is expected in regimes of CFT at GLR $=10 \%$, OIG and Y-jet at GLR $=5 \%$. In CFT and OIG, it should cause a change of the internal flow from the annular to the plug flow (Mlkvik et al., 2015). In the Y-jet atomizer, it should cause the liquid stream to penetrate into the centre of the mixing chamber, which results in the change of the liquid distribution within the chamber (Song and Lee, 1996).

Table 3. Liquid/gas momentum ratio $\Phi$ for examined regimes.

\begin{tabular}{|c|cccc|}
\hline \multirow{2}{*}{ Atomizer } & \multicolumn{4}{|c|}{ GLR [\%] } \\
& 2.5 & 5 & 10 & 20 \\
\hline CFT & 30.5 & 7.3 & 1.8 & 0.5 \\
OIG & 8.2 & 1.9 & 0.5 & 0.1 \\
OIL & 0.7 & 0.2 & 0.1 & 0.1 \\
Y-jet & 11.2 & 2.8 & 0.7 & 0.2 \\
\hline
\end{tabular}

Further increase of $\Phi$ (larger than 3) forces the liquid stream to penetrate into the mixing chamber at even higher velocities. These regimes are characteristic for low-GLR regimes, especially for CFT, OIG, and Y-jet atomizers. Under these internal conditions, we can expect a collision of the liquid fractions inside the mixing chamber and formation of the so-called plug flow in CFT and OIG atomizers. The Y-jet atomizer differs due to its specific construction as discussed below. 


\subsubsection{Description of the mixing processes in the selected atomizers}

This section deals with, the mixing process of each atomizer individually. The resulting assumptions of the internal two-phase flows are presented in Table 4.

CFT atomizer: the liquid streams enter the mixing chamber of the CFT atomizer perpendicularly to the main axis, see Fig. 1, d). Under the low-GLR regimes $(\Phi>3)$, the two-phase flow has a character of plug flow. Liquid streams penetrate into the mixing chamber and interact with each other and with air. Large liquid structures should be present but separated by large gas bubbles; the so-called plug flow. When the GLR increases ( $\Phi$ decreases) the gas bubbles enlarge and the internal flow should change from plug to annular.

OIL atomizer: the performance of this atomizer is influenced by a large fuel inlet area, 20 aerator holes, see Fig. 1 b), which results in a low velocity of the liquid as noted in Mlkvik et al., (2015) and discussed in Stähle et al., (2015a). This means that the liquid cannot penetrate into the centre of the mixing chamber, it flows on the walls of the chamber. Therefore, the value of $\Phi$ is relatively low and the annular flow is expected for all examined regimes.

OIG atomizer: The mixing mechanism in the OIG atomizer is almost opposite to the OIL atomizer; see Fig. $1 \mathrm{c}$ ). However, the formation of the annular internal flow occurs at low $\Phi$, i.e. high-GLR regimes; $10 \%$ and higher (Sovani et al., 2001). In the case of high $\Phi$ (low-GLR), the liquid momentum dominates. The gas should penetrate into the liquid stream through 20 aerator holes. However, a low gas momentum causes liquid penetration into the mixing chamber mainly through the rows close to the exit orifice (Stähle et al., 2015a). It causes the bubbles to flow near the walls of the chamber and interact when reaching the exit orifice. A collision of bubbles creates a local plug flow, which results in an unstable spray. This is in contradiction with standard effervescent atomization where a bubbly flow should be present (Sovani et al., 2001). However, recent studies (Mlkvik et al., 2015; Stähle et al., 2015a) show that at low-pressure regimes the liquid/gas momentum ratio plays a major role in the formation of the internal two-phase flow.

$Y$-jet atomizer: this type inherently forms an annular liquid film within the mixing chamber due to its specific construction (Mullinger and Chigier, 1974), see Fig. 1, a). The liquid stream enters the mixing chamber under the intersecting angle. Then the stream adheres to the cylindrical wall of the mixing chamber which creates an annular flow. It should be noted that the asymmetry and spatial development of the internal flow is influenced by the liquid/gas momentum ratio, as described in (Song and Lee, 1996). For regimes characterized by the low values of $\Phi$ (less than one) the liquid stream does not have sufficient momentum to penetrate fully to the opposite side of the mixing chamber. Thus it flows on the side where the liquid entered the chamber and then it spreads around the wall. When the momentum ratio fits within 1 and 3, the liquid should penetrate into the chamber and create a relatively well dispersed annular flow. Further increase of momentum ratio causes the liquid stream to reach the opposite wall and create again an annular flow. However, the emerging two-phase flow is asymmetric; the liquid film is thicker on the opposite side to the fuel port, as noted in Song and Lee, (1996).

Table 4. Resulting assumed internal flow regimes.

\begin{tabular}{|c|cccc|}
\hline \multirow{2}{*}{ Atomizer } & \multicolumn{4}{|c|}{ GLR [\%] } \\
& 2.5 & 5 & 10 & 20 \\
\hline CFT & Plug & Plug & Plug & Annular \\
OIG & Plug & Plug & Annular & Annular \\
OIL & Annular & Annular & Annular & Annular \\
Y-jet & Annular & Annular & Annular & Annular \\
\hline
\end{tabular}

\subsection{Link between internal flow and liquid discharge}

We used visualisation for the analysis of the near-nozzle area to link the information about the estimation of the internal flow and the character of the discharged liquid. Results of the internal flow 
estimation indicate the transition between flow regimes with varying GLR. Thus, the influence of a change in the internal flow is discussed in the following sections.

In this section, we neglect the propagation of pressure waves upstream through the atomizer, as was observed by Sen et al., (2014). These waves are generated by choking the exit orifice with large gas bubbles. The pressure waves might deform the gas bubbles upstream inside the flow. We assume that in the selected atomizers, the internal flows differ only in terms of the separated liquid and gas phases. Thus the pressure waves should not have a significant impact on the internal two-phase flow.

\subsubsection{Unstable flows}

Sprays generated by the internal-mixing twin-fluid atomizers are assumed to be inherently unstable. It was proved for the effervescent atomizers by Luong and Sojka, (1999). Here we define an unstable regime as a regime where two or more breakup mechanisms are present. At low-GLRs, we estimate the plug flow in OIG and CFT atomizers, as shown in Table 4. The plug flow is characterized by separated liquid volumes which are followed by large gas bubbles that result in a temporally varied GLR at the exit of the atomizer. This explanation is in agreement with visualisations of the liquid discharge since CFT and OIG atomizers produce unstable sprays. This is the reason why undisturbed liquid streams appear, see Fig. 4 c); these randomly switch into a well-atomized spray, see Fig 4. a), and vice versa. Meanwhile we also observed transition regimes, such a partial disruption of the liquid stream, see Fig 4. b). CFT is characterized by three different breakup regimes: bubble explosion, asymmetrical partial disruption and no disruption of the liquid stream, see Fig. 4. Similarly, in the case of OIG atomizer, four breakup regimes were observed at $2.5 \%$ and similarly at $5 \%$ of GLR: bubble explosion, large bubble explosion, partial disruption and no disruption of the liquid stream, see Fig. 5.

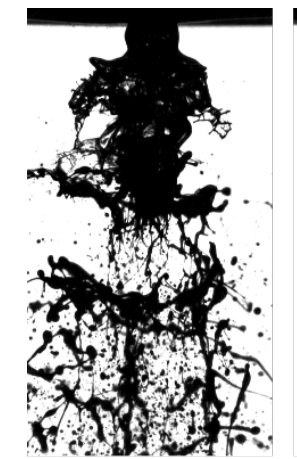

a)

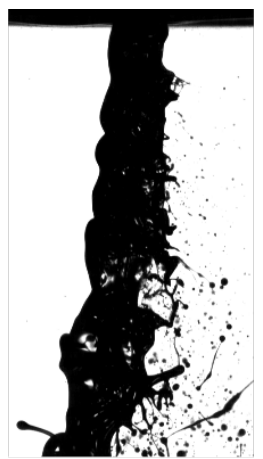

b)

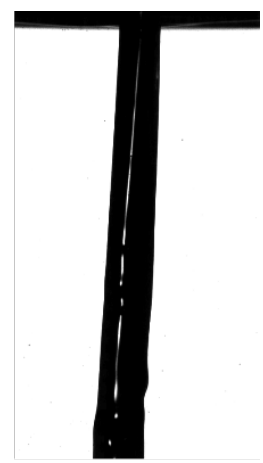

c)

Fig. 4 Emerging two-phase flow, CFT atomizer at GLR = 2.5\%: a) explosions of bubbles, b) asymmetrical partial disruption of the liquid stream, c) no disruption of the liquid stream.

According to Table 4, the CFT atomizer operated at GLR $=10 \%$ should produce a plug flow regime. However, we noticed that the breakup mechanism was relatively stable. It suggests that the internal two-phase flow reached the state where the liquid and gas were well mixed, which resulted in this behaviour. 


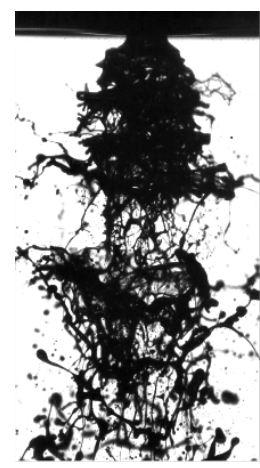

a)

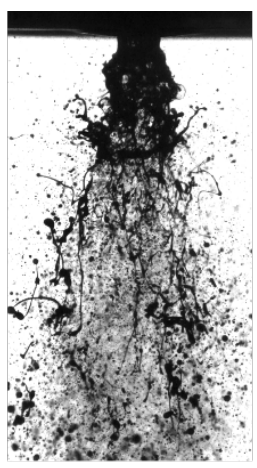

b)

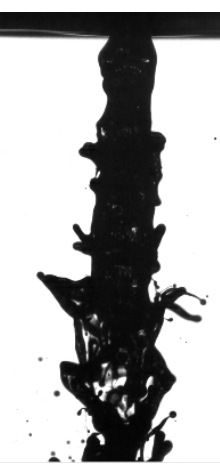

c)

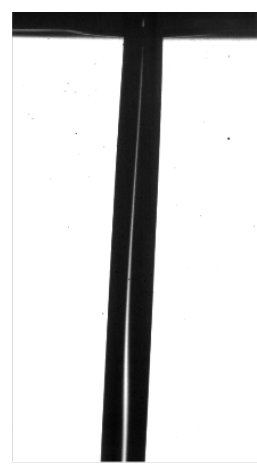

d)

Fig. 5 Emerging two-phase flow, OIG atomizer at $G L R=2.5 \%$ : a) explosions of bubbles, b) explosions of large bubbles, c) partial disruption of the liquid stream, d) no disruption of the liquid stream. Similar regimes were also observed for $G L R=5 \%$.

\subsubsection{Stable flows}

The OIL atomizer produces a relatively stable spray for all of the examined regimes. We identified only a single breakup regime for all investigated GLRs, i.e. the bubble explosion regime, see Fig 6 . However, assumptions of internal flow pattern (see Table 4) are not in agreement with the visualisations. The annular internal flow should result in the breakup mechanism that is similar to the disintegration of the annular liquid sheet (Santangelo and Sojka, 1995). Surprisingly, we observed that the liquid breakup works on the principle of bubble explosions which is characteristic of the effervescent atomization. This might be explained by internal flow observation by Stähle et al., (2015a). They described the spatial development of the internal two-phase flow in the mixing chamber. The annular-shaped liquid flows along the chamber downstream to the exit orifice of the atomizer. When it reaches the entrance to the exit orifice, the thickness of the liquid film enlarges, which allows the generation of instabilities; this in turn leads to the formation of liquid bridges. When these bridges are present, a local slug flow occurs which leads to the formation of the slug flow at the orifice discharge. Visualisations show that this process is quasi-periodical. This was also identified for OIG and CFT atomizers under assumed internal annular flow regimes.

The Y-jet atomizer differs demonstrably compared with the other selected atomizers. The assumed internal annular flow was observed for all regimes outside the atomizer. Due to a wider exit orifice, see Fig 1. a), this atomizer produces a slower two-phase mixture in the shape of an annular liquid sheet and a gas core inside. This liquid structure partly disrupts by the gas expansion (the first gas expansion occurs inside the mixing chamber). This statement is supported by both the raw recordings and the processed mean images where sprays, generated by this atomizer, are narrower compared to those of other atomizers. It suggests that the main momentum transfer between gas and liquid takes place in the mixing channel. This is the reason why the expansion in the radial direction is minimal comparing the other atomizers. Also, large liquid structures and ligaments appear even further downstream from the exit orifice.

\subsection{Quantitative Description of Liquid breakup}

We have made a series of near-nozzle area visualisations from the selected four atomizers (Fig 6.) For quantitative description, we examined the mean and RMS images obtained from a sequence of 100 snapshots for all regimes and selected atomizers. An increase of GLR leads to the generation of smaller liquid fragments (ligaments) and droplets, as expected (Sovani et al., 2001; Konstantinov et al., 2010). This applies to all the atomizers. The mean images give us an insight to the spray boundaries and relative density whereas the RMS images indicate the spatial variations in the series of snapshots as mentioned in section 3.2. The highest intensities in RMS images, red colour, show the regions with demonstrable differences between the individual images. These areas show a rapid extension of the liquid phase. The two intense red zones close the atomizers and symmetrical about the 
spray core, appear when the bubble expansion takes place. As the GLR ratio increases, these zones are then shortened and move closer to the atomizer. This was observed for the OIG, OIL and CFT atomizers under stable regimes.

\subsubsection{Unstable breakup regimes}

As mentioned in section 4.2.1, unstable regimes were observed for the OIG and CFT atomizers. The cause of this behaviour was the plug flow present inside the atomizer. In the mean figures, these regimes are characterized by the dark blue colour, undisturbed stream in the middle of the spray which indicates a transition among the breakup regimes, as can be seen in Figs. 4 and 5. At low-GLR regimes, it can be seen in CFT and OIG atomizers. RMS images (Fig. 8) show similar trends. For the CFT and OIG atomizers, there is again a dark blue area that indicates the undisturbed liquid stream. The expansion of liquid phase, red colour stripes, occurs further from the atomizer, and it has an oblong shape which is a manifestation of the partial disruption of the liquid stream, see Figs. 4 and 5.

\subsubsection{Stable breakup regimes}

In general, the blue zones, representing the densest parts of the spray, contract in the axial direction but expand in the radial direction as the GLR increases. A deformation of this area in the images is a consequence of the increasing amount of gas. Thus bubble explosions occur closer to the atomizer. In other words, the momentum ratio between the gas and liquid increases; therefore, the liquid disrupts earlier. Moreover, the thickness of the emerging liquid structures reduces with growing GLR. This means that the initial disturbances and deformations require less energy (Carvalho and Heitor, 1998; Wahono et al., 2008).

For the Y-jet atomizer, there are significant differences in the proceeded spray images. Both the mean and RMS images show that the spray is relatively narrow, short, and less dense. It appears that there are two vertical stripes. The presence of these stripes confirms that the annular liquid sheet penetrates downstream the nozzle, which supports the assumption of the internal flow documented in Table 4. The annular liquid sheet breaks up into large fragments, the so-called ligaments. This breakup occurs further from the atomizer due to a low discharge velocity that results in the generation of large droplets. From the mean images, we can see that the asymmetry of the spray varies with GLR. At $2.5 \%$ and $5 \%$ GLR, the spray is relatively symmetrical, which corresponds to the findings of Mlkvik et al., (2015) and Song and Lee, (1996). At higher GLR, the majority of the liquid is pushed to the right, a consequence of exceeding a certain level of liquid/gas momentum ratio as mentioned in the discussion on the internal flow estimation, section 4.1.1.

In general, CFT, OIG and OIL atomizers perform similarly except for the unstable regimes. The Yjet atomizer differs demonstrably. The liquid breakup is usually not finished even at the bottom part of the figure, which means that we can expect large liquid structures and droplets even further downstream from the atomizer. 


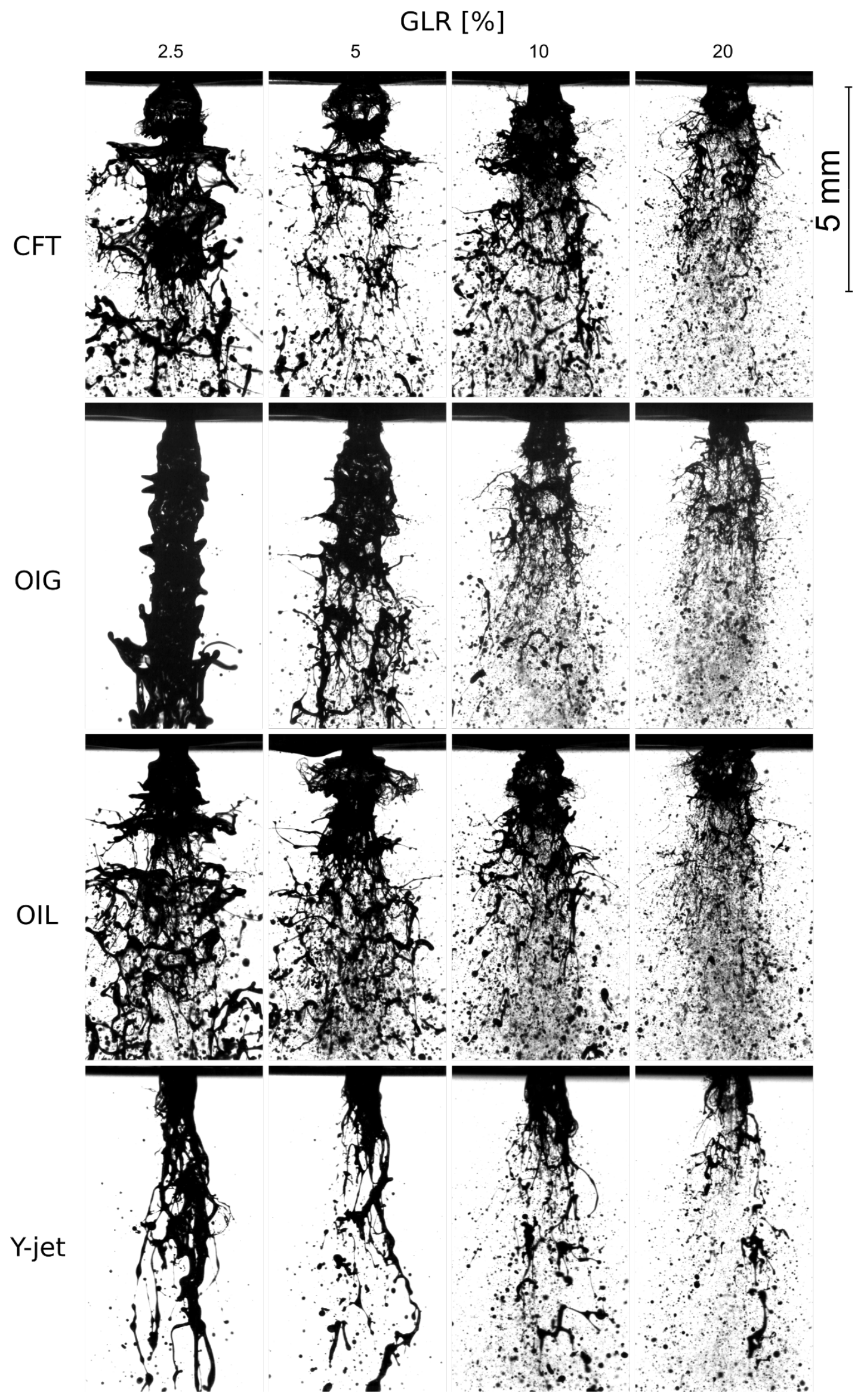

Fig. 6 Sample snapshots of liquid discharged from the atomizer. The exit orifice diameters are $0.7 \mathrm{~mm}$ for CFT, OIG and OIL and $1 \mathrm{~mm}$ for Y-jet atomizer, see Fig 1. 


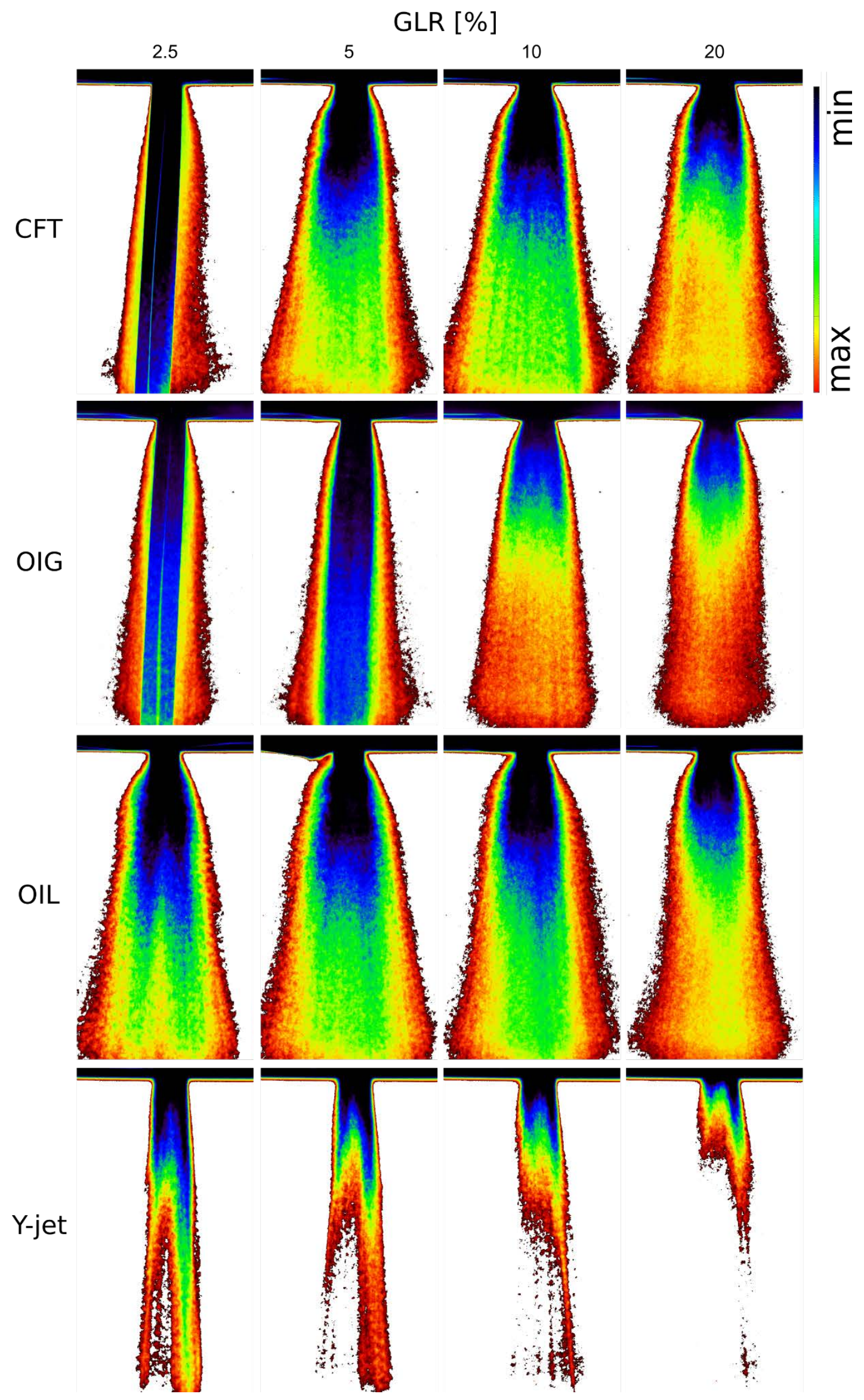

Fig. 7 Mean images obtained from 100 snapshots. 


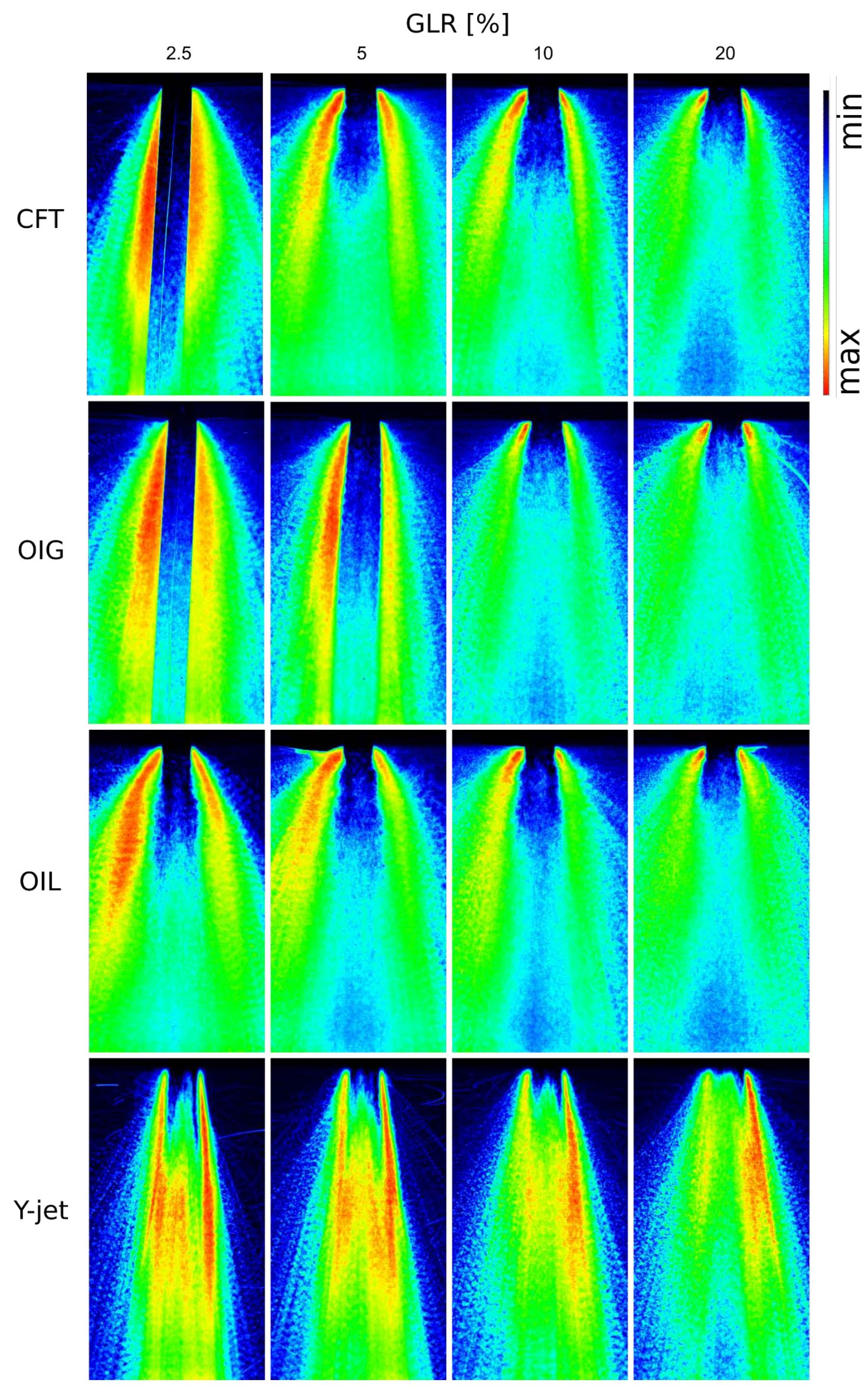

Fig. 8 RMS images obtained from 100 snapshots. 


\subsection{Droplet dynamics}

This section discusses the influence of GLR on the dynamics of droplets in the spray. Moreover, the characteristics of the spray are discussed concerning the internal mixing processes. PDA data were analysed to quantify the axial and radial droplet velocities as a relation of size.

\subsubsection{Influence of the GLR on droplets size-velocity distribution}

The mechanisms of the liquid breakup have a demonstrable influence on the downstream droplet dynamics (Jedelsky and Jicha, 2012). Thus we discuss the influence of the internal flow, consequent breakup mechanism and the resulting droplet size distribution.

During the liquid breakup, ligaments and large droplets are formed from the discharging liquid. In OIL, OIG, and CFT, the expansion of gas accelerates these liquid fragments in the near-nozzle area. This may cause their disintegration, i.e. the secondary breakup, which leads to the formation of individual droplets. These are further accelerated up to the speed of flowing gas. Small droplets reach the maximum velocity relatively quickly whereas the large ones require more time. When this process has finished, i.e. the breakup process, the large droplets maintain their velocity in the downstream axial direction from the orifice due to their relatively high momentum. In contrast, the small droplets decelerate together with the gas due to the interaction with the surrounding ambient atmosphere.
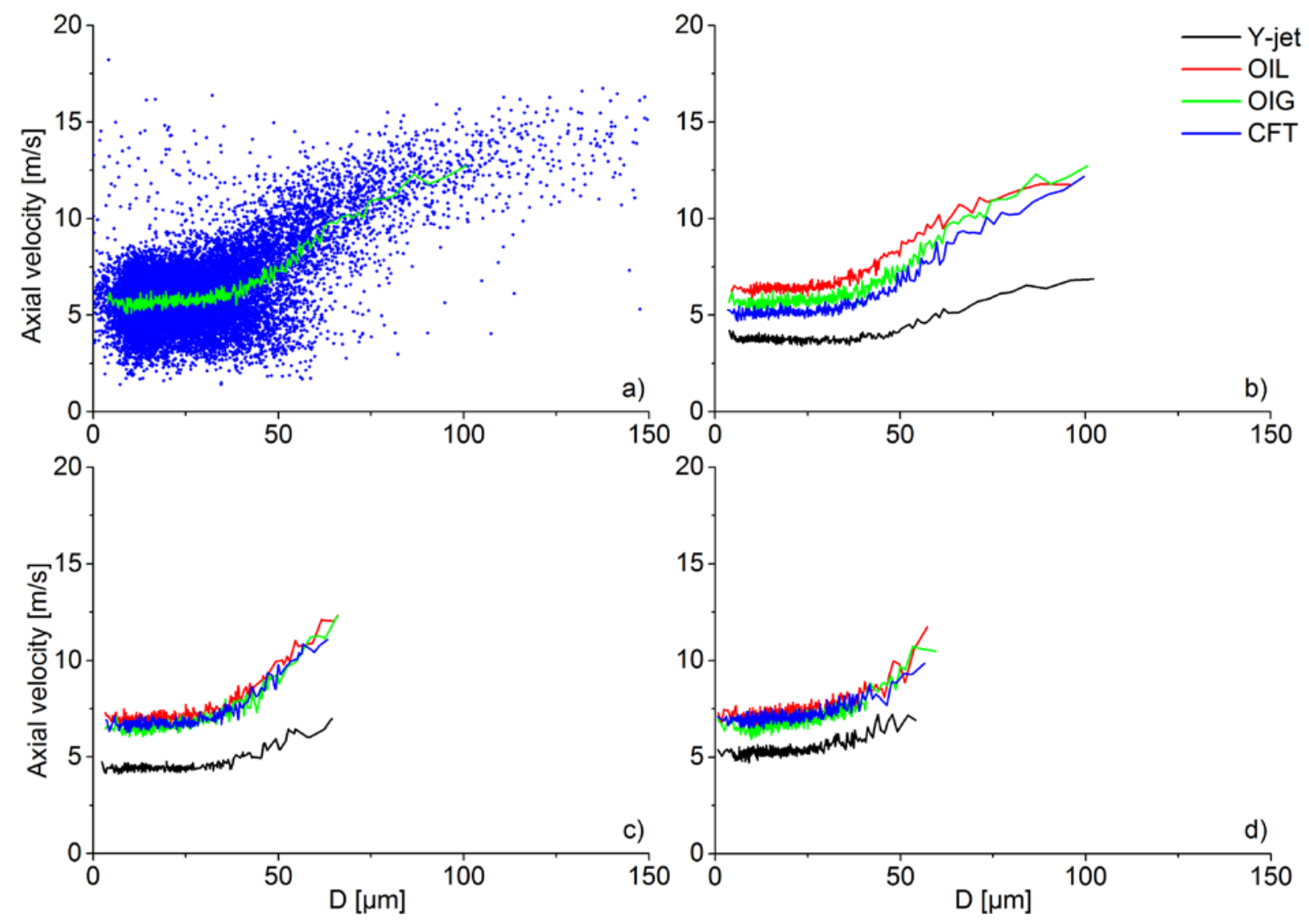

Fig. 9 Typical correlation between droplet size and axial velocity together with a floating average profiles for a point in the centre of the spray at the axial distance of $100 \mathrm{~mm}$ : a) sample distribution at $G L R=5 \%$, b) $G L R=$ $5 \%$, c) $G L R=10 \%$, d) $G L R=20 \%$.

A sample graph showing the axial velocity versus the droplet diameter for the measured droplets together with floating average is plotted in Fig. 9 a) for one measured point in the centre of the spray. In this graph, the majority of small droplets, up to ca. $50 \mu \mathrm{m}$, appear in the low-velocity area. For larger droplets, the size is positively correlated with the velocity. The maximum velocity was reached 
by the largest droplets with a diameter around $150 \mu \mathrm{m}$. A similar trend, often called the overshooting phenomenon (Lasheras, 1998), was observed for all atomizers. This behaviour is in agreement with previous findings (Jedelsky and Jicha, 2012) for the effervescent atomizers where the liquid breakup works by the bubble explosion regime. However, the overshooting phenomena manifests less in the Yjet atomizer due to the lower fluctuations in axial velocity, see Fig. 9.

The velocity floating averages are used to compare atomizers and operating regimes quantitatively, Figs. 9 b), c) and d). The OIL, OIG and CFT atomizers perform similarly, as expected. This is because these atomizers operate on similar breakup mechanism; the bubble explosions regime as mentioned in the previous section. The Y-jet atomizer differs, the floating average profile is flatter; thus the velocity fluctuations are smaller. Compared with other selected atomizers, the Y-jet atomizer inherently generates a relatively wide annular liquid sheet which has a consistent air core in the mixing chamber and the near-nozzle region. By introducing the liquid and gas into the mixing chamber, these fluids experience a partial momentum exchange. Due to the expansion of the gas, the liquid film accelerates and the velocity of liquid and gas phase partly equalize. This means that no bubble explosions occur, and only small fluctuations in droplet velocity were noted. The differences between the Y-jet atomizer and other atomizers decrease with the increase of GLR, which might be attributed to the transition from various internal flows into the annular flow, which was assumed for all high-GLR regimes.

For the CFT, OIL and OIG atomizers, stable breakup regimes are characterized by the bubble explosions mechanism. As the GLR increases the bubbles are enlarged and the frequency of bursts increases as well. It results in the generation of thinner liquid fractions and small droplets. Thus the resulting droplet size distribution is narrower, which leads to smaller velocity variations. This trend is clearly seen in Fig. 9 where the increasing GLR causes a reduction in the velocity fluctuations.
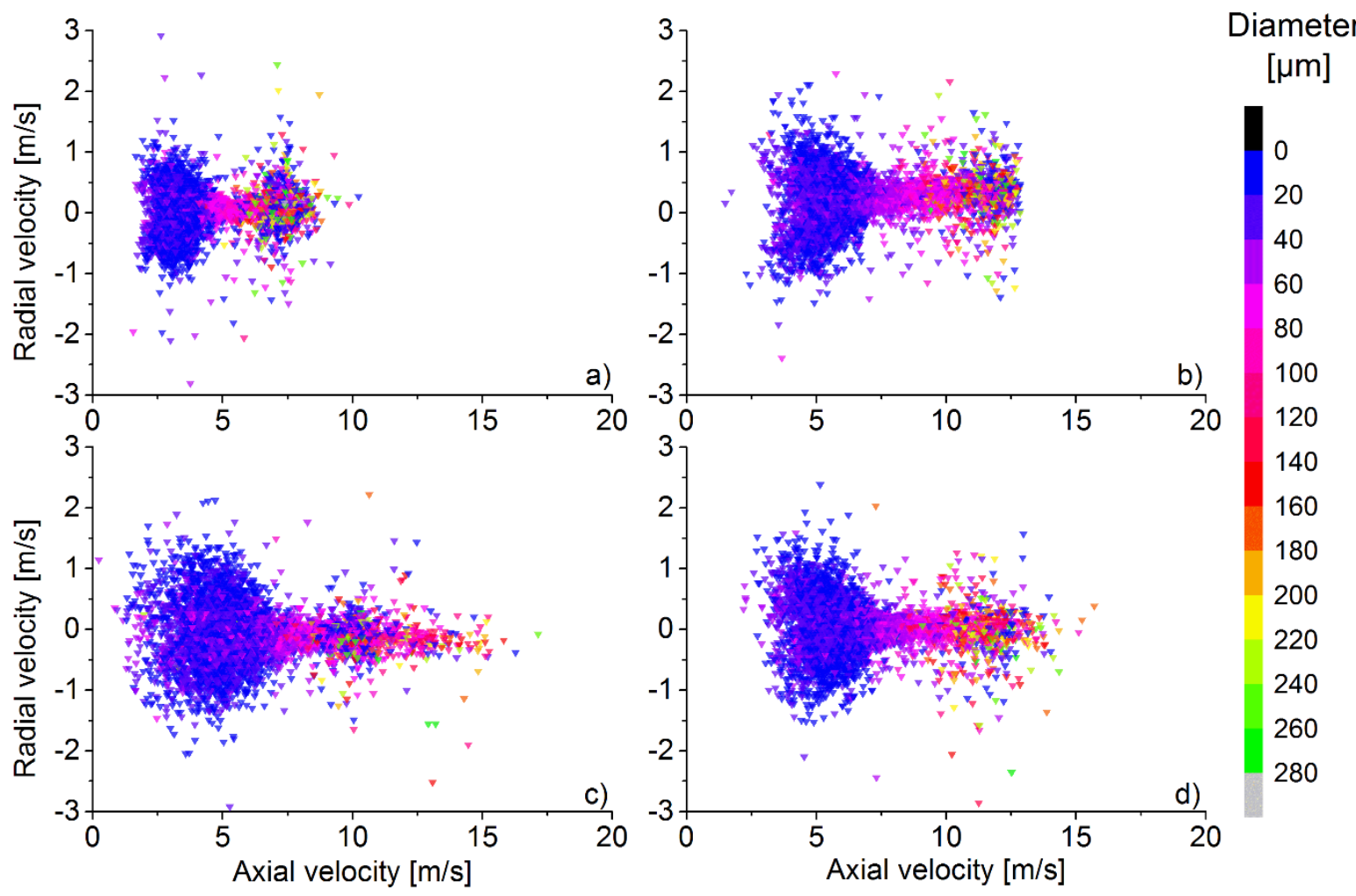

Fig. 10 Size/velocity correlation in the centre of the spray at GLR $=2.5 \%$. Only 5,000 samples are shown for better visibility: a) Y-jet, b) OIL, c) OIG, d) CFT.

The velocity/size correlations including both the radial and axial velocity component for $G L R=2.5 \%$ are shown in Fig. 10 where we observed the largest differences among the atomizers. Two significant clouds of droplets can be seen for all the atomizers. The first cloud, in the lower axial 
velocity area, is characterized by a wide span of radial velocities. The droplets have a diameter from 0 up to 50 microns and typically reach the values of $S t k<0.1$. It means that they easily follow the gas flow (Jedelsky and Jicha, 2012).

The second characteristic particle cloud emerges in the high axial velocity area (higher than $5 \mathrm{~m} / \mathrm{s}$ in Y-jet atomizer and higher than $7 \mathrm{~m} / \mathrm{s}$ in others). It is characterized by an increase of radial velocity and occupied by droplets of all sizes, i.e. from small droplets, of 5 microns up to 280 microns. The majority of these droplets are created during a gas pulse from bubble explosions.
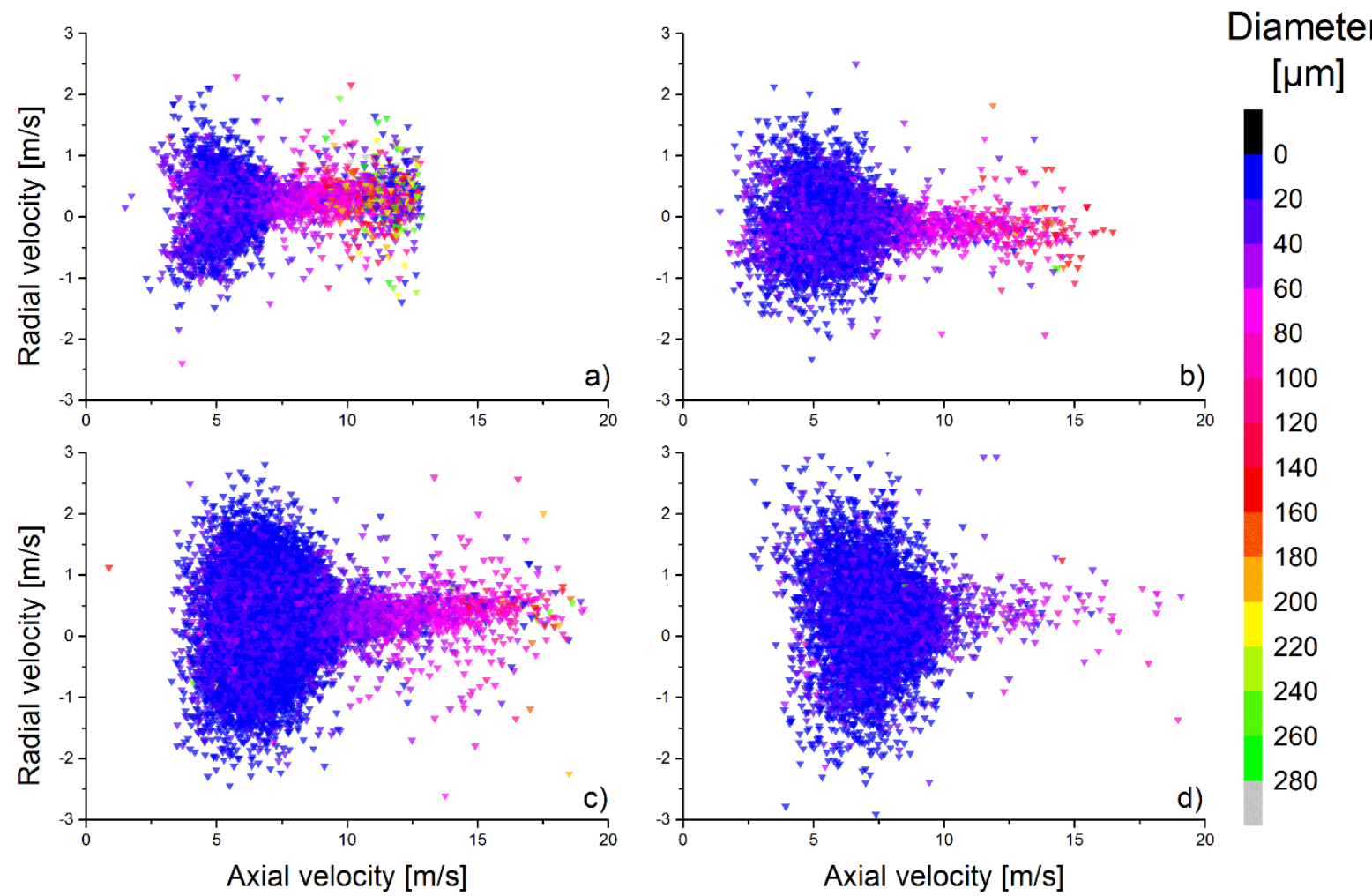

Fig. 111 Size/velocity correlation of OIL atomizer obtained in the centre of the spray. Only 5,000 samples are shown for better visibility: a) $G L R=2.5 \%$, b) $G L R=5 \%$, c) $G L R=10 \%$, d) $G L R=20 \%$.

For the OIL atomizer, the influence of the operating regime on the droplet size/velocity correlations is shown in Fig. 11. Similar distributions were also found for the four of atomizers. The bi-modal distribution (presented at $G L R=2.5 \%$ ) is transferred into distribution with even stronger dependency of droplet size on the axial velocity, regimes at 5 and $10 \%$ of GLR, see Fig. 11 . Moreover, the amount of the largest droplets, green colour, is rapidly reduced with the increase in GLR. At GLR $=5 \%$, there is a nearly straight line represented mainly by droplets sized from 50 to 100 microns. At $G L R=10 \%$, the area expands to even higher axial velocities. Further increase of GLR causes a reduction of this area, which corresponds to the decrease in the mean droplet size. Small droplets cannot reach such high velocities at the examined axial distance due to their low momentum, i.e. their velocity is affected by drag forces. We have plotted only one relationship for the OIL atomizer but similar trends, as shown in Fig. 11, are observed for the other atomizers, for $G L R=5 \%$ and higher. The differences among atomizers are reduced as GLR grows. This is in agreement with the transition of the internal flow from the plug flow to the annular flow, which occurs for all atomizers at high-GLR regimes. From the above, it can be concluded that the internal design of the atomizer influences droplet dynamics mainly for low-GLR regimes. 


\subsubsection{The interaction between droplets}

In the previous section, the velocity-size distributions were discussed. It should be noted that there are some relatively small droplets which have much higher velocity than is the average for a given droplet size range.

This behaviour can be explained by plotting the time-resolved data of the droplet velocity and size measurements. Fast and large droplets, larger than 100 microns, mainly for $G L R=2.5 \%$, are frequently accompanied by droplets smaller than 30 microns, of velocity higher than the mean velocity for a given size range. This can be seen in Fig. 12 at the times of 50, 67 and 97 milliseconds. These events occur approximately ten times per second in one measured point, taken from the sample of 500 droplets from each atomizer. The number of such events is rapidly reduced with the increase in GLR. It is assumed that the relatively small and fast droplets were influenced by the large ones. One of possible explanations is that the large droplets entrain the small ones due to local vortices.

When the particle exceeds a certain value of Reynolds number, higher than 100, vortices should occur behind this particle (Gushchin and Matyushin, 2006). Then the droplets with very low Stokes numbers might be entrained behind the large droplet. However, it is necessary to keep in mind that all calculations, which predict the droplets behaviour, are simplified, and the gas velocity was not measured directly. Note that the data allow a temporal resolution of 0.1 microseconds.

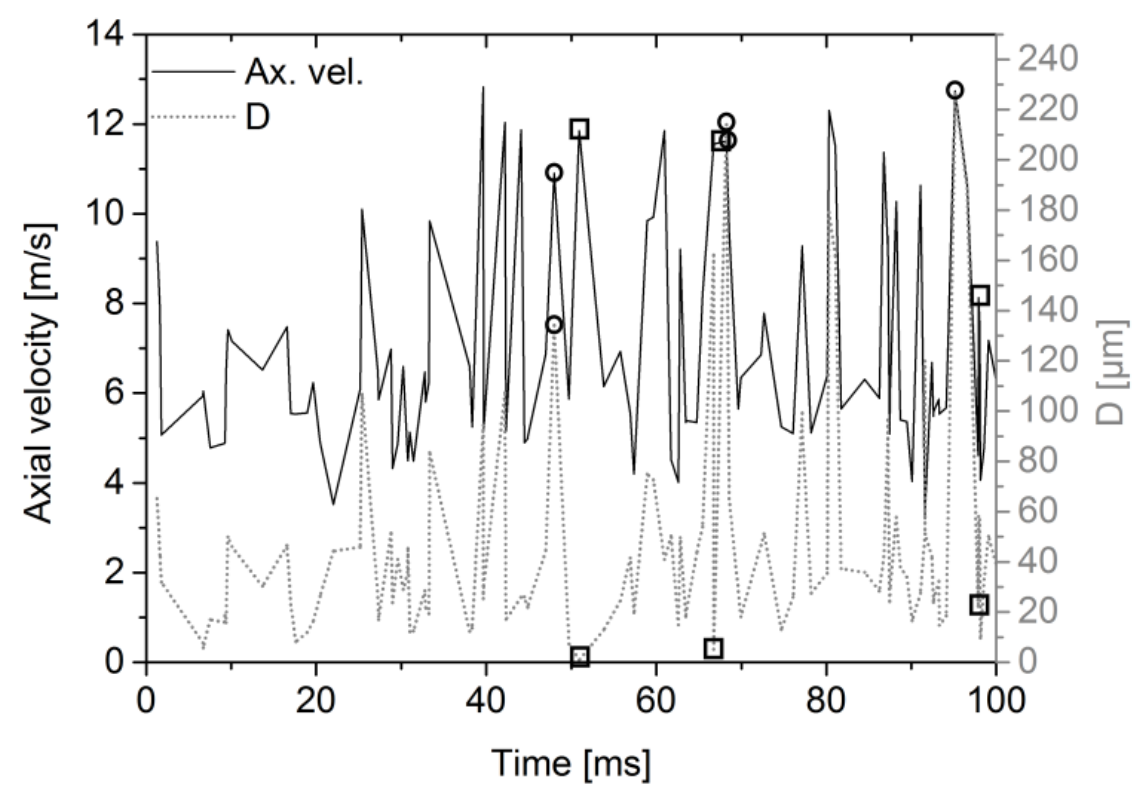

Fig. 122 Time-resolved data of droplet size and axial velocity for OIL atomizer at GLR $=2.5 \%$. Black squares mark the velocity and size of small and fast droplets whereas black circles mark large and fast droplets.

\subsection{Droplet size distribution}

Using the PDA data, the local Weber numbers were calculated. It was found that $98 \%$ of measured droplets had Weber numbers lower than its critical value (11 in our case). This suggests that in the measured points, 15 points in the entire radial profile at the axial distance of $100 \mathrm{~mm}$, the majority of atomization processes were completed. However, visualizations showed that even at this axial distance, large non-spherical liquid structures were present at unstable regimes of CFT and OIG atomizers. Since the PDA system can obtain relevant information only from spherical droplets, we do not consider the measurements of droplet size at these regimes; the results showed suspiciously low values of mean droplet size. This is because the large non-spherical liquid fragments contain majority of liquid mass which the PDA system cannot take into account. Except for the large liquid structures, the sprays contained a well-atomized liquid, produced by the breakup mechanism similar to that in Fig. 4 a) or Fig. 5b). This was the cause of the low values of mean droplet size. 
The droplet size distribution was evaluated using an integral value of Sauter mean diameter (ID32), see Table 5. As expected, GLR negatively correlates with droplet size for all regimes and all atomizers. The low operating pressure and relatively high liquid viscosity caused that the ID32 values were systematically larger compared to the previous results (Avulapati, 2012). The Y-jet atomizer produces the largest droplets for all regimes, which is in agreement with the visualisations of the liquid breakup. The CFT and OIG atomizers perform similarly. A relatively stable spray of good quality was generated by the OIL atomizer. This atomizer generates the spray of lowest ID32 for the regimes from 2.5 to $10 \%$ of GLR.

Table 5. Values of ID32 for examined operating regimes.

\begin{tabular}{|c|cccc|}
\hline \multirow{2}{*}{ Atomizer } & \multicolumn{4}{|c|}{ GLR [\%] } \\
& 2.5 & 5 & 10 & 20 \\
\hline CFT & - & 81.9 & 63.2 & 56.4 \\
OIG & - & - & 63.2 & 54.7 \\
OIL & 100.4 & 70.3 & 62.9 & 57.8 \\
Y-jet & 127.1 & 99.6 & 78.7 & 65.1 \\
\hline
\end{tabular}

\section{Conclusions}

Four different twin-fluid atomizers have been studied and compared in this paper. The spray formation was analysed starting with the estimation of an internal flow in the atomizer, followed by the description of the liquid breakup, up to the analysis of droplet motion in the region of fully developed spray. The results point out the importance of the internal design of the atomizer, especially under the low-GLR, high-efficient regimes. Moreover, the internal design influences the whole process of the spray formation. From the present study, several concluding remarks can be drawn:

- Atomizers of a certain design, OIL or Y-jet, inherently create an annular internal flow that generates a relatively stable spray for a wide range of GLR.

- OIG and CFT atomizers operate with plug flow at low-GLR, which results in an unstable and poorly atomized spray.

- The droplet dynamics differs demonstrably between the atomizers for low-GLR regimes. We can attribute this behaviour to the differences in the internal two-phase flows. The difference in size/velocity correlations further reduces when GLR increases, which corresponds to the transition of various internal flows into an annular flow pattern.

- The spray behaviour points to the interaction of droplets in the region of the fully developed spray. The droplets larger than 100 microns might entrain and drag along small droplets locally.

- It was shown that the Phase Doppler analyser cannot obtain relevant results of mean drop size of the unstable sprays where large non-spherical structures were presented.

- The mean droplet size is negatively correlated with GLR as expected. The largest droplets were produced by the Y-jet atomizer; this was confirmed by the visualizations. The CFT, OIG, and OIL atomizers have similar droplet size distributions. However, only the OIL atomizer was able to generate a relatively stable spray for all examined regimes.

- When assuming the application in the combustion process, the OIL atomizer is the only one capable of working under the examined range of low-pressure and low-GLR regimes. Using the Y-jet atomizer, higher operating pressures are needed to achieve a fine spray. The CFT and OIG atomizers must operate with an annular internal flow to generate a stable spray under selected operating pressure. 


\section{Acknowledgements}

The authors gratefully acknowledge the financial support from the project No. GA15-09040S funded by the Czech Science Foundation and financial support of the European Regional Development Fund within the framework of Operational Programme "'Research and Development for Innovations" NETME Centre TechUp project, reg. nr. CZ.1.05/2.1.00/19.0397. Authors express their thanks to Dr. Graham Wigley for the contribution to the PDA data analysis and the grammar corrections. 


\section{References}

Avulapati, Madan Mohan, and R. V. Ravikrishna. "An Experimental Study on Effervescent Atomization of Bio-Oil Fuels." Atomization and Sprays 22, no. 8 (2012): 663-85.

Bayvel, L.P. and Orzechowski, Z., "Liquid Atomization." Taylor \& Francis, Washington, (1993).

Buckner, Harry N., and Paul E. Sojka. "Effervescent Atomization of High-Viscosity Fluids: Part I. Newtonian Liquids." Atomization and Sprays 1, no. 3 (1991): 239-52.

Carvalho, I. S., and M. V. Heitor. "Liquid Film Break-up in a Model of a Prefilming Airblast Nozzle."Experiments in Fluids 24, no. 5-6 (May 1998): 408-15.

Catlin, C. A., and J. Swithenbank. "Physical Processes Influencing Effervescent Atomizer Performance in the Slug and Annular Flow Regimes." Atomization and Sprays 11, no. 5 (Sep-Oct 2001): 575-95.

Chin, J.S., "Effervescent atomization and internal mixing air assisted atomization." Int. J. Turbo Jet Engines 12, (1995): 119-127.

Diego-Marin, A, C Melendez-Cervantes, AAM Aranda, and A Giles-Alarcon. "Improvement of the Performance of a Utility Oil Fired Boiler by Modifying the Design of Burners and Atomizers." Asme Power Conference 2009 (2009): 107-13.

Dumouchel, Christophe. "On the Experimental Investigation on Primary Atomization of Liquid Streams." Experiments in Fluids 45, no. 3 (Sep 2008): 371-422.

Eggers, J., and E. Villermaux. "Physics of Liquid Jets." Reports on Progress in Physics 71, no. 3 (Mar 2008): 79.

Ferreira, M.E., Teixeira, J.C.F., Bates, C.J., Bowen, P.J., "Detailed investigation of the influence of fluid viscosity on the performance characteristics of plain-orifice effervescent atomizers." Atomization Sprays 11, 2001.

Ferreira, G., J. A. Garciia, F. Barreras, A. Lozano, and E. Lincheta. "Design Optimization of TwinFluid Atomizers with an Internal Mixing Chamber for Heavy Fuel Oils." Fuel Processing Technology 90, no. 2 (Feb 2009): 270-78.

Gadgil, HP, and BN Raghunandan. "Effect of Air Injection Configuration on Characteristics of Effervescent Sprays." Journal of Propulsion and Power 27, no. 4 (Jul-Aug 2011): 925-28.

Gadgil, Hrishikesh P., and B. N. Raghunandan. "Some Features of Spray Breakup in Effervescent Atomizers." Experiments in Fluids 50, no. 2 (Feb 2011): 329-38.

Ghaemi, S., P. Rahimi, and D. S. Nobes. "Effect of Bubble Generation Characteristics on Effervescent Atomization at Low Gas-Liquid Ratio Operation." Atomization and Sprays 20, no. 3 (2010): 21125.

Gushchin, V. A., and R. V. Matyushin. "Vortex Formation Mechanisms in the Wake Behind a Sphere for $200<\operatorname{Re}<380$." Fluid Dynamics 41, no. 5 (2006): 795-809.

Huang, X., X. Wang, and G. Liao. "Visualization of Two Phase Flow inside an Effervescent Atomizer." Journal of Visualization 11, no. 4 (Oct 2008): 299-308.

Jedelsky, J., and M. Jicha. "Droplet Dynamics in Internally Mixed Twin-Fluid Spray." Paper presented at the WIT Transactions on Engineering Sciences, (2014).

Jedelsky, J, and M Jicha. "Energy Conversion During Effervescent Atomization." Fuel 111 (Sep2013): 836-44.

Jedelsky, Jan, and Miroslav Jicha. "Spatially and Temporally Resolved Distributions of Liquid in an Effervescent Spray." Atomization and Sprays 22, no. 7 (2012): 603-26.

Jedelsky, J, and M Jicha. "Unsteadiness in Effervescent Sprays: A New Evaluation Method and the Influence of Operational Conditions." Atomization and Sprays 18, no. 1 (2008): 49-83.

Jedelsky, J, M Jicha, J Slama, and J Otahal. "Development of an Effervescent Atomizer for Industrial Burners." Energy \& Fuels 23 (Dec 2009): 6121-30. 
Jedelský, J., M. Zaremba, M. Malý, and M. Jícha. "Characteristics of Droplet Motion in Effervescent Sprays." Paper presented at the EPJ Web of Conferences, (2014).

Ju, DH, JH Fang, TT Zhang, XQ Qiao, J Xiao, and Z Huang. "High-Speed Shadow Imaging in Internal Flow Pattern and Macroscopic Characteristics of a R134a Flash-Boiling Spray Discharged through a Vertical Twin-Orifice Atomizer." International Journal of Multiphase Flow 75 (Oct 2015): 224-36.

Konstantinov, Dancho, Richard Marsh, Phil Bowen, and Andrew Crayford. "Effervescent Atomization for Industrial Energy-Technology Review." Atomization and Sprays 20, no. 6 (2010 2010): 525-52.

Li, Z. H., Y. X. Wu, C. R. Cai, H. Zhang, Y. L. Gong, K. Takeno, K. Hashiguchi, and J. F. Lu. "Mixing and Atomization Characteristics in an Internal-Mixing Twin-Fluid Atomizer." Fuel 97 (Jul 2012): 306-14.

Liu, M., Y. Duan, T. Zhang, and Y. Xu. "Evaluation of Unsteadiness in Effervescent Sprays by Analysis of Droplet Arrival Statistics - the Influence of Fluids Properties and Atomizer Internal Design." Experimental Thermal and Fluid Science 35, no. 1 (2011): 190-98.

Lund, M. T., Paul E. Sojka, Arthur H. Lefebvre, and P. G. Gosselin. "Effervescent Atomization at Low Mass Flow Rates. Part I: The Influence of Surface Tension." Atomization and Sprays 3, no. 1 (1993): 77-89.

Luong, J. T. K., and P. E. Sojka. "Unsteadiness in Effervescent Sprays." Atomization and Sprays 9, no. 1 (1999): 87-109.

Mlkvik, M., P. Stähle, H. P. Schuchmann, V. Gaukel, J. Jedelsky, and M. Jicha. "Twin-Fluid Atomization of Viscous Liquids: The Effect of Atomizer Construction on Breakup Process, Spray Stability and Droplet Size." International Journal of Multiphase Flow 77 (2015): 19-31.

Mujumdar, A. S., L. X. Huang, and X. D. Chen. "An Overview of the Recent Advances in SprayDrying." Dairy Science \& Technology 90, no. 2-3 (Mar-Jun 2010): 211-24.

Mullinger, P. J., and N. A. Chigier. "The Design and Performance of Internal Mixing Multijet Twin Fluid Atomizers." J. Inst. Fuel 47, no. 393 (1974): 251-61.

Pacifico, Antonio L., and Jurandir I. Yanagihara. "The Influence of Geometrical and Operational Parameters on Y-Jet Atomizers Performance." Journal of the Brazilian Society of Mechanical Sciences and Engineering 36, no. 1 (Jan 2014): 13-22.

Santangelo, P. J., and P. E. Sojka. "A Holographic Investigation of the near-Nozzle Structure of an Effervescent Atomizer-Produced Spray." Atomization and Sprays 5, no. 2 (1995): 137-55.

Santolaya, JL, LA Aisa, E Calvo, I Garcia, and JA Garcia. "Analysis by Droplet Size Classes of the Liquid Flow Structure in a Pressure Swirl Hollow Cone Spray." Chemical Engineering and Processing 49, no. 1 (Jan 2010): 125-31.

Sen, D., M. A. Balzan, D. S. Nobes, and B. A. Fleck. "Bubble Formation and Flow Instability in an Effervescent Atomizer." Journal of Visualization 17, no. 2 (2014): 113-22.

Song, SH, and SY Lee. "Study of Atomization Mechanism of Gas/Liquid Mixtures Flowing through Y-Jet Atomizers." Atomization and Sprays 6, no. 2 (MAR-APR 1996 1996): 193-209.

Sovani, S. D., P. E. Sojka, and A. H. Lefebvre. "Effervescent Atomization." Progress in Energy and Combustion Science 27, no. 4 (2001): 483-521.

Stähle, P., V. Gaukel, and H. P. Schuchmann. "Investigation on the Applicability of the Effervescent Atomizer in Spray Drying of Foods: Influence of Liquid Viscosity on Nozzle Internal Two-Phase Flow and Spray Characteristics." Journal of Food Process Engineering 38, no. 5 (Oct 2015): 47487.

Stähle, Philipp, Heike P. Schuchmann, and Volker Gaukel. "Performance and Efficiency of PressureSwirl and Twin-Fluid Nozzles Spraying Food Liquids with Varying Viscosity." Journal of Food Process Engineering (2015): n/a-n/a.

Sutherland, J. J., P. E. Sojka, and M. W. Plesniak. "Ligament-Controlled Effervescent Atomization." Atomization and Sprays 7, no. 4 (Jul-Aug 1997): 383-406.

Tamaki, N., Shimizu, M., Hiroyasu, H., "Atomization of high viscous liquid jet by internal mixing twin-fluid atomizer. " In: ILASS Europe, (2004): pp. 412-417 
Wahono, Stevano, Damon Honnery, Julio Soria, and Jamil Ghojel. "High-Speed Visualisation of Primary Break-up of an Annular Liquid Sheet." Experiments in Fluids 44, no. 3 (Mar 2008): 45159. 